\title{
O processamento de lexemas com combinação afixal múltipla no português europeu ${ }^{1}$
}

\author{
Alexandra Soares Rodrigues \\ Escola Superior de Educação- Instituto Politécnico de Bragança \\ CELGA-ILTEC- Universidade de Coimbra
}

\begin{abstract}
:
This work aims to understand the limits to the syntagmatic extension of multiple derivational affixation which deals with affixes that operate in productive word-formation patterns of contemporary European Portuguese (RioTorto et al., 2016). The work is theoretically supported on the multiple-route model (Kuperman et al., 2010) and empirically based on the analysis of corpora (Corpus de Referência do Português Contemporâneo and Linguateca) and of experiments (acceptability judgement task and recall task) made with native speakers of European Portuguese. The empirical phenomenon of the multiple affixation under focus is constituted by lexemes containing the suffix series -bil/-al-iz(a)-bil-idade, which is observable in words with low frequency such as comercializabilidade and materializabilidade. Under the perspective of structural constraints (Gaeta, 2015; Rodrigues, 2014; 2015), multiple affixation should be possible as long as the constraints between affixes are obeyed. However, the low frequency of the multiple affixation under analysis arouses the hypothesis that processual conditionings influence the limits of multiple affixation. The acceptability judgement task and the recall task aim to shed light on the psycholinguistic conditions that intervene in the limits of multiple affixation. The study concludes that variables such as the frequency of the affix combination, semantic transparency, affix salience, and expectedness (Bell \& Schäffer, 2013; 2016) towards the combination are important for the pattern construction power of the affix combination, that is, for the capacity of the combination to function as a mental pattern that allows the speaker to produce and analyze lexemes. The pattern construction power depends on the experience of the speaker towards the affix combination.
\end{abstract}

Keywords: multiple affixation, word-formation patterns, processing conditions, structural constraints, Portuguese. Palavras-chave: afixação múltipla, padrões genolexicais, condições processuais, constrangimentos estruturais, português.

\section{Introdução}

\subsection{Notas sobre o trabalho}

O presente trabalho tem como objetivo a análise da interação entre restrições estruturais e condições processuais que limitam a extensão da combinação afixal. Este trabalho apresenta complementaridade com Rodrigues (em publicação; 2016).

\footnotetext{
${ }^{1}$ Gostaríamos de agradecer aos revisores anónimos os seus cometários e sugestões que permitiram melhorar o texto.
}

№ 3 - 09/ 2017 | 285-310 | https://doi.org/ 10.26334/2183-9077/rapln3ano2017a16 
Expõe-se aqui uma investigação que tem por base nomes em -bil/-al-iz(a)-bil-idade, encontrados em edições hodiernas de periódicos de Portugal (e.g. Público) e em buscas na internet. Trata-se de uma séria afixal com baixa representatividade (um total de 563 ocorrências no Google e na Linguateca; frequência de 0/milhão de palavras, de acordo com o Corpus de Referência do Português Contemporâneo (CRPC)).

Nessa investigação fez-se a comparação entre o processamento de

i) nomes em -bill-al-iz(a)-bil-idade, ou seja, de palavras com afixação múltipla heterocategorial com baixa frequência ou geradas ad hoc (e.g. materializabilidade, contabilizabilidade);

ii) palavras com afixação múltipla heterocategorial de uso mais frequente do que a categoria anterior (média de frequências de 2,33/milhão de palavras, de cordo com o CRPC) (e.g. ornamentalização, desintoxicação);

iii) nomes com afixação isocategorial de tipo avaliativo de uso frequente (média de frequências de 2,85, de acordo com o CRPC) (e.g. pequenininho, cafezinho);

iv) nomes com afixação múltipla isocategorial de tipo avaliativo com baixa frequência ou gerados ad hoc (frequência de 0/milhão de palavras, de acordo com o CRPC) (e.g. mesinhitazinha, gatinhazinha);

v) pseudopalavras, criadas ad hoc (e.g. *quirilete, *vontoco), e

vi) palavras neutras (frequentes (média de frequência de 235/milhão de palavras), básicas ou com estrutura morfológica derivacional sem correlação com aquelas que são alvo do estudo) (e.g. espacial, paciência).

As pseudopalavras e as palavras neutras foram usadas com o objetivo de funcionarem como ponto de comparação com os lexemas contendo as estruturas visadas, mas também como distratores. Enquanto distratores, estas palavras obstam que o participante nas tarefas de recordação construa respostas com base em predições resultantes da constatação de regularidades morfológicas entre os estímulos.

O nosso confronto empírico com nomes em -bil/-al-iz(a)-bil-idade suscitou uma série de questões que nos conduziu à investigação exposta em Rodrigues (em publicação; 2016). Essas questões, que procedem umas das outras e que a seguir se apresentam segundo a ordem dessa procedência, constituem os cernes das secções do nosso trabalho: 
a) Os nomes que contêm a série afixal -bil/-al-iz(a)-bil-idade são representativos de uma combinação afixal frequente em português (secção 2)?

Se não, como foi comprovado através do confronto dos itens e da combinatória afixal em corpora (Linguateca, Corpus de Referência do Português Contemporâneo) e no Google,

b) estes nomes encontram-se estruturalmente bem construídos, obedecendo a combinação afixal em causa a constrangimentos estruturais?

Se sim, como verificaremos na secção 3,

c) embora sejam pouco frequentes, serão considerados como aceitáveis no português europeu pelos falantes nativos (secção 4)?

d) os falantes apresentarão o mesmo nível de aceitação face a outras combinações afixais de carácter mais frequente (secção 4)?

Se não, como a experiência 1 (secção 4) comprovou,

e) que motivações suportarão a maioritária classificação como 'não aceitável' por parte dos falantes nativos e a baixa frequência dos itens lexicais (pressupondo haver uma relação entre ambas) (secção 5)?

A questão e) motivou a experiência 2 (secção 5), que consistiu em tarefas de recordação.

A análise dos resultados conduziu a outras questões:

f) A combinação afixal -bill-al-iz(a)-bil-idade constitui-se como um padrão derivacional do português (secções 6 e 7)?

$\mathrm{E}$, finalmente,

g) Que consequências decorrerão para o processamento lexical de itens complexos construídos com uma combinação afixal não coincidente com um padrão derivacional (secções $6,7$ e 8$) ?$

A nossa investigação conclui que, embora a série afixal -bil/-al-iz(a)-bil-idade obedeça a constrangimentos estruturais, não constitui um padrão genolexical, uma vez que não cumpre condições processuais necessárias. Essas condições são a frequência, a previsibilidade da combinatória, a transparência da mesma e a saliência afixal.

Para procedermos à construção das respostas a f) e g), é necessário partir de conceptualizações respeitantes ao processamento das palavras complexas, a que dedicaremos de modo breve (por razões de espaço) a próxima secção. Uma discussão mais aprofundada da 
mesma, com referência a outros trabalhos pertinentes, pode ser consultada em Rodrigues (em publicação).

\subsection{Modelos e fatores determinantes do processamento de palavras complexas}

A representação mental e o processamento das palavras complexas têm sido motivação para diferentes modelos (Schreuder \& Baayen, 1995; Baayen, 2007; Libben, 1998; Bertram et al., (2000), Kuperman et al., 2010).

Um dos modelos clássicos de tipo single-route, o modelo sublexical, consiste numa abordagem computacional ou de decomposição morfológica da palavra complexa (Taft, 1979; 1981; Andrews, 1986; Andrews et al., 2004; Marslen-Wilson, et al. 1994). Outro modelo clássico, também ele de tipo single-route, o modelo supralexical, sugere que estas palavras são mentalmente representadas e armazenadas como uma unidade em bloco (Butterworth, 1983; Giraudo \& Grainger, 2001). Sendo assim, segundo o modelo supralexical, no processamento das palavras complexas não ocorre decomposição nos seus constituintes morfológicos.

Para além da complexidade morfológica da palavra, outros fatores intervêm no modo de processamento dos lexemas complexos. Esta observação suscitou a emergência de outros modelos, tais como os modelos dual-route. De acordo com estes modelos, a frequência é um desses fatores. Assim, a via direta é mais eficiente em palavras complexas de elevada frequência, devido ao fator da frequência, (Lehtonen et al., 2007; Frauenfelder \& Schreuder, 1991; Schreuder \& Baayen, 1995; Baayen et al., 1997). Segundo os modelos dual-route, pode ocorrer competição entre as duas vias de acesso, saindo vencedora aquela para a qual ocorrerem mais fatores que a favoreçam.

Sem que nos dediquemos à explicitação e análise dos modelos em causa, destacamos apenas que têm sido assinalados outros fatores como a frequência do lexema, a categoria lexical, o seu carácter derivacional, flexional ou composto, as relações semânticas entre as palavras e os seus constituintes morfológicos (Libben, 1998:31), a frequência da base e do radical (Taft \& Forster, 1976; Beauvillain, 1996; Burani \& Caramazza, 1987; Schreuder et al., 2003; Niswander et al., 2000; Niswander-Klement \& Pollatsek, 2006), a frequência da forma da palavra (Baayen et al., 2007), a transparência semântica (Marslen-Wilson et al., 1994; Libben et al., 2003; Bell \& Schäffer, 2013; 2016), a produtividade (Baayen, 1992; 1993), a saliência afixal (Laudanna \& Burani, 1995), entre outros. 
Todos esses fatores devem ser tidos em consideração quer na edificação de modelos quer na análise do processamento das palavras complexas. Esse reconhecimento conduziu à formulação de modelos como o de Kuperman et al. (2008, 2009, 2010), o modelo multipleroute.

Kuperman et al. (2010:94) concebem o léxico mental como "long-term memory storage for lexical information", sendo que "The ease of access, and generally of lexical processing, depends in part on the amounts of information carried by words, which are defined by the accumulated knowledge of words and their paradigmatic and syntagmatic connectivity in the mental lexicon. The multiple-route model considers morphological structure as a conglomerate of sources of information, which contribute - to a different extent - to the recognition of polymorphemic words".

É seguindo esta abordagem que, no nosso estudo, tomaremos em consideração os fatores frequência da combinação afixal, transparência semântica, saliência afixal, previsibilidade da combinação afixal, complexidade morfológica dos padrões genolexicais e a capacidade de construção de padrão. De acordo com Bell \& Schäffer (2013, 2016), quanto maior for a frequência, maior é a previsibilidade e, logo, maior é a transparência semântica.

A previsibilidade e a capacidade de construção de padrão estão dependentes de informação na memória com a qual o estímulo possa ser identificado. Só assim poderá ocorrer reconhecimento ou da palavra ou do padrão de que a palavra é instância. De acordo com o nosso estudo, essa informação na memória não se restringe a informação relativa a morfemas ou até a regras ou esquemas onde operam esses morfemas. É necessária também informação acerca das combinações entre os morfemas não apenas um a um, mas multiplamente. A nossa perspetiva está de acordo com Caramazza et al. (1998), Schreuder \& Baayen (1995), Frauenfelder \& Schreuder (1991) e Ji et al. (2011), que demonstram que o processamento das palavras complexas necessita de um procedimento não apenas de decomposição das mesmas nos seus constituintes, mas também de um procedimento integrativo dos constituintes no seu todo. Neste procedimento, a transparência semântica tem um papel decisivo, como demonstrado por Libben et al. (2003: 51).

Nas palavras em -bil-/-al-izabilidade, não obstante a composicionalidade do seu significado, este não resulta transparente. Como observaremos, tal deve-se ao facto de os afixos que compõem a combinatória não serem salientes, visto que a combinação afixal envolvida não 
é previsível, uma vez que não é frequente. É pois determinante a experiência do falante face à combinação afixal em jogo. Estamos, pois, de acordo com Bell \& Schäfer (2016: 163), no seu estudo sobre compostos, que referem que "[...] the perceived transparency is correlated both with the expectedness of the constituents themselves and with the expectedness of this relation". No nosso estudo, verificamos que a combinatória -bil/-al-izabilidade, ainda que obedeça a constrangimentos estruturais e que possua um significado componencial, não resulta semanticamente transparente. Tal acontece pelo facto de a combinatória não ser previsível, não sendo reconhecida pelo falante como um padrão.

Fatores como frequência da combinação, previsibilidade e transparência semântica determinam aquilo que designamos por capacidade de construção de padrão. Esta consiste na capacidade de uma combinação afixal funcionar como um padrão mental que serve a construção e a análise de palavras complexas. Essa capacidade depende da experiência do falante.

Expostos brevemente os conceitos básicos inerentes à compreensão dos fatores intervenientes no processamento das palavras complexas, descreveremos em seguida o nosso estudo.

\section{Frequência da combinação afixal -bil/-al-iz(a)-bil-idade}

Na secção 1.1, expusemos a seguinte pergunta (a):

Os nomes que contêm a série afixal -bill-al-iz(a)-bil-idade são representativos de uma combinação afixal frequente em português?

A análise de corpora (Linguateca e Corpus de Referência do Português Contemporâneo) e a procura em motores de busca da internet (Google) devolve uma resposta negativa em relação à frequência destas palavras complexas. As buscas no Google e na Linguateca devolveram, em conjunto, apenas 563 ocorrências. O CRPC aponta uma frequência de $0 /$ milhão de palavras.

Tendo em conta os dados relativos à sua frequência, procedemos em seguida à averiguação da questão (b) anunciada na secção 1.1:

Estes nomes encontram-se estruturalmente bem construídos, obedecendo a combinação afixal em causa a constrangimentos estruturais? 


\section{Adequação a constrangimentos estruturais}

Sob uma perspetiva lexicalista, a formação de palavras é encarada como sendo regulada por padrões que têm sido descritos e explicados de acordo com diversos modelos. Alguns modelos equacionam esses padrões como regras de formação de palavras (Halle, 1973; Aronoff, 1976; Corbin, 1987; Rio-Torto, 1993); outros como esquemas (Booij, 2010). Não sendo objetivo deste trabalho a explanação desses modelos, remetemos para Baeskow (2015) para uma panorâmica acerca dos mesmos.

Os modelos de regras de formação de palavras e de esquemas concebem a existência de constrangimentos estruturais que determinam a adequação entre afixos e bases ou entre bases e mecanismos genolexicais não afixais, como é o caso da conversão (Rodrigues, 2009), aos níveis de diversas fiadas estruturais fonológicas, semânticas e sintáticas e das suas interfaces (Rainer, 2005; Rodrigues, 2009; 2014; 2015). Estes constrangimentos impedem que, por exemplo, num processo de afixação, qualquer afixo operador numa dada regra de formação de palavras se anexe a qualquer base da mesma regra. Por limitações de tempo/espaço, não especificaremos aqui os tipos de constrangimentos estruturais existentes, limitando-nos a demonstrar a adequabilidade da combinação -bil/-al-iz(a)-bil-idade a esse nível.

A afixação múltipla compreendida em -bill-al-iz(a)-bil-idade obedece a constrangimentos estruturais previstos em padrões genolexicais expostos nas Tabelas 1 e 2:

\begin{tabular}{|c|c|c|c|}
\hline $\begin{array}{l}\text { Padrão/Combinatória } \\
\text { 1a }\end{array}$ & Padrão/Combinatória 2 & $\begin{array}{l}\text { Padrão/Combinatória } \\
3\end{array}$ & $\begin{array}{l}\text { ??Padrão??/Combinatóri } \\
\text { a } 4\end{array}$ \\
\hline $\begin{array}{l}\text { Adjetivos relacionais } \\
\text { denominais }\end{array}$ & Verbos deac & $\begin{array}{l}\text { Adjetivos modais } \\
\text { deverbais }\end{array}$ & Nomes de qualidade \\
\hline $\begin{array}{l}\text { Base }_{\text {Nsimples }} \\
\text { Adjetivo -al }\end{array}$ & Verbo & $\begin{array}{l}\text { Base }_{\mathrm{V}-i z_{-}}>\text {Adjetivo - } \\
\text { vel }\end{array}$ & $\begin{array}{l}\text { Base }_{\mathrm{ADJ}-v e l}>\text { Nome - } \\
\text { idade }\end{array}$ \\
\hline 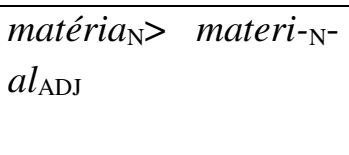 & $\begin{array}{l}\text { material }_{\mathrm{ADJ}}>\text { material }_{\mathrm{ADJ}} \\
-i z-\mathrm{v}\end{array}$ & 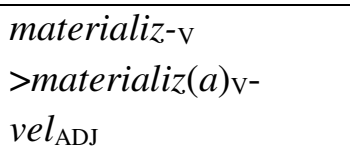 & $\begin{array}{l}\text { materializável }_{\mathrm{ADJ}} \\
>\quad \text { materializabil-ADJ- } \\
\text { idade }_{\mathrm{N}}\end{array}$ \\
\hline $\begin{array}{l}\text { comércio }_{\mathrm{N}}> \\
\text { comerci }_{-\mathrm{N}}-a_{\mathrm{ADJ}}\end{array}$ & 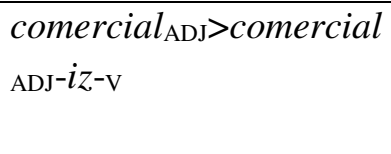 & $\begin{array}{l}\text { comercializ-v } \\
>\text { comercializ }(a)_{\mathrm{V}^{-}} \\
\text {vel }_{\mathrm{ADJ}}\end{array}$ & $\begin{array}{l}\text { comercializável }_{\mathrm{ADJ}}> \\
\text { comercializabil }_{\mathrm{ADJ}-} \\
\text { idade }_{\mathrm{N}}\end{array}$ \\
\hline
\end{tabular}

Tabela 1. Padrões/combinatórias envolvidas na afixação múltipla de palavras em -al-iz(a)-bil-idade 
Na Tabela 1, podemos observar que a combinatória 1a corresponde ao padrão genolexical segundo o qual é possível gerar adjetivos denominais relacionais através do afixo -al, a partir de um nome morfologicamente não complexo. A combinatória 2 mostra o padrão segundo o qual se podem construir verbos com o sufixo -iz-, partindo de adjetivos em -al. A combinatória 3 coincide com o padrão que permite a construção de adjetivos modais com o sufixo - vel a partir de bases verbais com o sufixo -iz-. A combinatória 4, que neste trabalho avaliaremos como não coincidente com um padrão genolexical, é instância da possibilidade de formação de nomes em -idade a partir de adjetivos em -vel. Os lexemas apontados nas tabelas 1 e 2 até à combinatória 3, inclusive, encontram-se atestados em dicionários como o da Infopédia (www.infopedia.pt) e o Aulete (www.aulete.br).

\begin{tabular}{|c|c|c|c|}
\hline $\begin{array}{l}\text { Padrão/Combinatória } \\
1 \mathrm{~b}\end{array}$ & $\begin{array}{l}\text { Padrão/Combinatória } \\
2\end{array}$ & $\begin{array}{l}\text { Padrão/Combinatória } \\
3\end{array}$ & $\begin{array}{l}\text { ??Padrão??/Combinatória } \\
4\end{array}$ \\
\hline $\begin{array}{l}\text { Adjetivos modais } \\
\text { deverbais }\end{array}$ & Verbos deadjetivais & $\begin{array}{l}\text { Adjetivos modais } \\
\text { deverbais }\end{array}$ & Nomes de qualidade \\
\hline $\begin{array}{l}\text { Base vsimples > Adjetivo } \\
\text {-vel }\end{array}$ & $\begin{array}{l}\text { Base }_{\mathrm{ADJ}-v e l}>\text { Verbo - } \\
i z-\end{array}$ & $\begin{array}{l}\text { Base }_{\mathrm{V}-i z-}>\text { Adjetivo - } \\
\text { vel }\end{array}$ & $\begin{array}{l}\text { Base }_{\mathrm{ADJ}-v e l}>\text { Nome - } \\
\text { idade }\end{array}$ \\
\hline $\begin{array}{l}\text { conta }-\mathrm{v}_{\text {conta }_{\mathrm{v}}-\text { vel }_{\mathrm{ADJ}}}>\end{array}$ & $\begin{array}{l}\text { contável }_{\mathrm{ADJ}} \\
>\text { contabil-}{ }_{\mathrm{ADJ}}-i z-\mathrm{v}\end{array}$ & 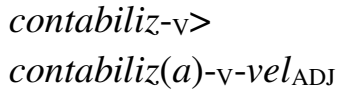 & 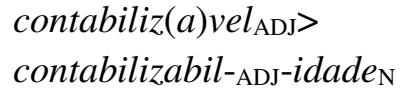 \\
\hline
\end{tabular}

Tabela 2. Padrões/combinatórias envolvidas na afixação múltipla de palavras em -bil-iz(a)-bil-idade

$\mathrm{Na}$ Tabela 2, o padrão $1 \mathrm{~b}$ prevê a formação de adjetivos modais em -vel a partir de bases verbais morfologicamente não complexas. Os restantes padrões são partilhados com a Tabela 1.

Na Tabela 3 mostramos $a b$ absurdum combinatórias que desobedeceriam a constrangimentos estruturais:

\begin{tabular}{|c|c|c|c|}
\hline PCombinatória 1 & PCombinatória 2 & PCombinatória 3 & ?Combinatória 4 \\
\hline $\begin{array}{l}\text { Adjetivos modais } \\
\text { deverbais }\end{array}$ & Verbos deadjetivais & $\begin{array}{l}\text { Adjetivos modais } \\
\text { deverbais }\end{array}$ & Nomes de qualidade \\
\hline $\begin{array}{l}{ }^{*} \text { Base }_{\mathrm{v}}>\text { Adjetivo - } \\
\text { ense }\end{array}$ & $\begin{array}{l}{ }^{*} \text { Base }_{\text {ADJ-ense }}>\text { Verbo - } \\
i z-\end{array}$ & $\begin{array}{l}* \text { Base }_{\mathrm{V}-\mathrm{iz}-}>\text { Adjetivo } \\
-u d(o)\end{array}$ & $\begin{array}{l}\text { * }^{\text {Base }} \text { ADJ-udo }>\text { Nome - } \\
\text { idade }\end{array}$ \\
\hline $\begin{array}{l}* \text { cont }-\mathrm{v}> \\
\text { cont }-\mathrm{v}-e n s(e)_{\mathrm{ADJ}}>\end{array}$ & $\begin{array}{l}* \text { contense } e_{\mathrm{ADJ}}>\text { contens }- \\
\mathrm{ADJ}^{-} i z-\mathrm{v}\end{array}$ & $\begin{array}{l}*_{\text {contabiliz-v }>} \\
\text { contabiliz-v-ud(o })_{\mathrm{ADJ}}\end{array}$ & $\begin{array}{l}{ }^{*} \text { contabilizudo }{ }_{\mathrm{ADJ}}> \\
\text { contabilizud }-{ }_{\mathrm{ADJ}}-{ }_{\text {idade }}\end{array}$ \\
\hline
\end{tabular}

Tabela 3. Hipotéticas combinatórias afixais não obedecentes a constrangimentos estruturais 
$\mathrm{Na}$ Tabela 3, colocámos afixos que operam em regras de formação de palavras. Contudo, anexámos esses afixos a bases que i) não pertencem a essa Regra ou ii), ainda que pertencentes a essa Regra, não admitem esses operadores afixais específicos. Assim, o sufixo -ens(e) é operador na formação de adjetivos, mas não de adjetivos modais, pelo que não se anexa a verbos. O sufixo -iz- labora na formação de verbos a partir de adjetivos, mas não de adjetivos sufixados em -ens(e). O sufixo $-u d(o)$ permite a construção de adjetivos, mas não a partir de verbos e, logo, não a partir de bases que contenham -iz-, que só podem ser verbos. $\mathrm{O}$ sufixo -idad(e) gera nomes de qualidade a partir de adjetivos relacionais, mas não a partir de adjetivos relacionais sufixados em $-u d(o)$.

Não sendo este o lugar para a explicitação das motivações desses constrangimentos, fica, pois, estabelecida a obediência a constrangimentos estruturais das combinações -bil/-aliz(a)-bil-idade.

A combinatorialidade afixal deve ser analisada em dois níveis: i) o nível de afixo por afixo; ii) o nível de combinatória múltipla das combinatórias afixo por afixo.

As Tabelas 1 e 2 mostram possíveis os dois níveis de combinatorialidade. Contudo, o que se verifica é que a combinatória 4 não resulta frequente e, por isso, pode não consistir num padrão genolexical.

Analisaremos em seguida as condicionantes processuais dessas combinatórias.

\section{Aceitação ou não pelos falantes nativos - Experiência 1}

A necessidade de avaliar a aceitabilidade de itens contendo a sequência afixal -bil/-al$i z(a)$-bil-idade e a sua comparação com outros itens contendo afixação múltipla mais frequente foram conducentes da Experiência 1. A Experiência 1 consistiu numa tarefa de julgamento de aceitabilidade que a seguir se descreve.

\subsection{Metodologia da Experiência 1}

A Experiência 1, coincidente com uma tarefa de julgamento de aceitabilidade, foi arquitetada com o objetivo de avaliar a aceitabilidade dos lexemas em -bil/-al-iz(a)-bil-idade, em confronto com as restantes categorias de lexemas apresentadas na secção 1, por parte dos falantes nativos do português europeu. 


\subsubsection{Participantes}

Os participantes da tarefa de julgamento de aceitabilidade constituíram o designado Grupo A. Integraram o Grupo A 22 falantes nativos do português europeu, com audição normal e visão normal ou corrigida até ao normal, estudantes do Instituto Politécnico de Bragança, sem conhecimentos de linguística.

\subsubsection{Estímulos}

Foram fornecidas em suporte escrito 86 palavras isoladas pertencentes às seguintes categorias: i) 27 palavras neutras (e.g. livro, pão); ii) 12 pseudopalavras (e.g. quirilete, arcadilo); iii) 13 nomes com afixação isocategorial de tipo avaliativo frequentes (e.g. cafezinho, passarinho); iv) 13 nomes/adjetivos com afixação múltipla de tipo avaliativo não frequentes ou gerados ad hoc para a experiência e seguindo os padrões derivacionais da categoria anterior (e.g. gatinhazinha, menininhazinha); v) 10 nomes terminados na série afixal-bil/-al-izabilidade (e.g. estabilizabilidade, socializabilidade. Dado que os nomes com esta sequência são pouco frequentes, foram criados ad hoc nomes seguindo essa sequência de afixos, para além daqueles que constam em corpora. Note-se que o importante não é verificar se um dado lexema se encontra atestado, mas se a combinatória afixal derivacional que o gera funciona efetivamente como um padrão mental); vi) 10 palavras heterocategoriais contendo pelo menos 3 afixos derivacionais (e.g. desgovernamentalização, compartimentalização).

No cômputo total, o número de palavras contendo a série -bil/-al-izabilidade é inferior aos das palavras avaliativas, uma vez que não se pretendia fazer saliente a ocorrência desta série afixal. Por outro lado, queria-se que o número destas palavras igualasse o número de outras palavras heterocategoriais, de modo a que a sua comparação fosse equitativa. O número de palavras neutras pretendia-se superior de maneira que as combinatórias afixais avaliativas e heterocategoriais se tornassem diluídas para os participantes.

\subsubsection{Procedimento}

As 86 palavras que constituíam os estímulos foram fornecidas ao Grupo A em papel. Os participantes tinham que assinalar se classificavam cada um dos estímulos como 'aceitável em português' ou 'não aceitável em português'. 


\subsection{Resultados da tarefa de julgamento de aceitabilidade}

Recordamos que, nesta exposição, apenas nos centraremos na comparação entre os nomes contendo a série afixal -bil/-al-izabilidade e palavras complexas com sufixação múltipla heterocategorial frequentes. Como está patente na tabela 4, os resultados da tarefa de julgamento de aceitabilidade apontam para uma tendência, por parte dos falantes nativos do português europeu contemporâneo, para classificarem como aceitáveis em português os lexemas frequentes por oposição àqueles que, não obstante serem igualmente construídos por afixação múltipla, são não frequentes.

\begin{tabular}{|l|r|r|}
\hline \multicolumn{1}{|c|}{ Estímulos } & $\begin{array}{l}\text { aceitável em } \\
\text { português }\end{array}$ & $\begin{array}{l}\text { não aceitável em } \\
\text { português }\end{array}$ \\
\hline Palavras em -bil/-al-izabilidade & $38 \%$ & $62 \%$ \\
\hline Palavras complexas heterocategoriais frequentes & $64 \%$ & $36 \%$ \\
\hline
\end{tabular}

Tabela 4. Resultados da tarefa de julgamento de aceitabilidade

Os resultados obtidos na tarefa de julgamento de aceitabilidade mostram que a frequência da palavra e da série afixal constituinte da palavra tem um efeito na aceitação por parte dos falantes dos itens em análise.

Tal como previsível pela série de questões anotadas na secção 1.1, perante a tendente não aceitação dos lexemas em -bil/-al-izabilidade por parte dos participantes, a nossa investigação direcionou-se para a questão e) que aqui repetimos:

Que motivações suportarão a maioritária classificação como 'não aceitável' por parte dos falantes nativos e a baixa frequência dos itens lexicais (pressupondo haver uma relação entre ambas)?

Esta questão conduziu-nos à Experiência 2, que a seguir se descreve.

\section{Motivações para a tendente não aceitação de nomes em -bil/-al-izabilidade - Experiência 2}

As motivações psicolinguísticas que influem na aceitabilidade deste tipo de lexemas podem ser averiguadas através de tarefas de recordação, que compreendem a Experiência 2. 


\subsection{Metodologia da Experiência 2}

\subsubsection{Participantes}

Os participantes da tarefa de recordação constituíram o designado Grupo B, constituído por 22 falantes nativos do português europeu, com audição normal e visão normal ou corrigida até ao normal, estudantes do Instituto Politécnico de Bragança, sem conhecimentos de linguística. Os participantes do Grupo A e os do Grupo B não são coincidentes.

\subsubsection{Estímulos}

Os mesmos lexemas descritos em 4.1 .2 a propósito da tarefa de julgamento de aceitabilidade serviram de estímulos na tarefa de recordação. No entanto, enquanto na primeira tarefa os lexemas foram apresentados isoladamente, na segunda, cada lexema foi inserido num enunciado. Cada enunciado tinha no máximo duas orações. Nestas condições, uma das orações era relativa. De outro modo, o enunciado dispunha apenas de uma oração. Cada enunciado só poderia conter um adjunto, sendo um sintagma adverbial ou preposicional. Os lexemas contidos nos enunciados que não eram as palavras-alvo tinham carácter frequente ou pertenciam a um tipo morfológico frequente. As palavras-alvo não apareciam no final dos enunciados. A duração mínima dos enunciados com as palavras neutras era, em segundos, de 2.00 s; a máxima de 4.82 s. A duração mínima dos enunciados com as pseudopalavras era de $2.00 \mathrm{~s}$; a máxima de $3.12 \mathrm{~s}$. A duração mínima de enunciados contendo palavras com a série -bil/-al-izabilidade era de 2.76 s; a máxima de 4.17 s. Nos enunciados com palavras heterocategoriais frequentes a duração mínima era de $2.77 \mathrm{~s}$; a máxima de 4.53 s. Nos enunciados com avaliativos frequentes, a duração mínima era de $1.82 \mathrm{~s}$; a máxima de $3.38 \mathrm{~s}$. Nos enunciados com avaliativos não frequentes, a duração mínima era de $1.99 \mathrm{~s}$; a máxima de $3.03 \mathrm{~s}$.

\subsubsection{Procedimento}

Apresentaram-se ao Grupo B 86 enunciados, cada um contendo um dos 86 lexemas já descritos em 4.1.2., em formato sonoro, numa gravação feita por um falante nativo de português europeu.

Os enunciados encontravam-se ordenados aleatoriamente. A tarefa dos participantes consistia em reproduzirem oralmente os enunciados, imediatamente após a sua audição. Optámos por inserir as palavras-alvo em enunciados para que a atenção do participante não 
recaísse imediatamente sobre a palavra-alvo e para que a receção das mesmas fosse feita num ambiente mais próximo da realidade do uso da língua, em que as palavras são usadas em enunciados e não isoladamente.

As produções obtidas, que foram gravadas, foram classificadas por nós em 7 classes distintas. Seria redundante e pouco pertinente operar somente com duas categorias iniciais (acertos e não acertos), para posteriormente trabalharmos com as 7 classes, uma vez que as duas categorias (acertos e não acertos) não nos dariam informação relevante acerca dos resultados obtidos.

Dado que o estudo incide especificamente sobre o processamento de lexemas e não dos enunciados, essas classes referem-se somente às produções dos lexemas e não àquelas dos enunciados na sua totalidade.

São as seguintes as classes das produções obtidas:

i) Acerto Lexical Completo (ALC): O lexema foi repetido corretamente.

ii) Mutação Fonológica Parcial (MFP): A estrutura fonológica foi alterada parcialmente de maneira que a forma produzida não é coincidente com um lexema real ou potencial do português (e.g. *famarialização por familiarizabilidade).

iii) Acerto Semântico Total (AST): Não tendo ocorrido repetição do lexema, houve produção de um lexema, real ou potencial, semanticamente equivalente àquele que constituía o estímulo. $\mathrm{O}$ acerto semântico total pode ocorrer devido a) à utilização de lexema sinónimo (e.g. também por igualmente); b) à manutenção do morfema lexical e mudança dos morfemas derivacionais por outros sinónimos (e.g. cadelinha por cadelinhazinha; lapinhozinho por lapinhozito. Os exemplos dados não se referem aos estímulos das categorias em foco neste texto, uma vez que não se obteve esta classe de produções para as duas categorias de lexemas analisadas neste trabalho.).

iv) Alteração Semântica Parcial (ASP): Não tendo ocorrido repetição do mesmo lexema, observou-se produção de lexema, real ou potencial, semanticamente equivalente àquele que constituía o estímulo apenas parcialmente. A equivalência entre os lexemas por vezes é apenas semântica e outras vezes semântica e formal. Para as categorias de lexemas em foco neste texto, este tipo de produção resulta da manutenção do morfema lexical e coocorrente mudança de morfemas derivacionais (e.g. globalização por globalizabilidade). 
v) Desacerto na Categoria Lexical (DCL): Ainda que fonologicamente o significante tenha sido repetido convenientemente, a forma produzida foi inserida num contexto sintático distinto daquele que caberia à categoria lexical do estímulo. (Não se verificou nas duas categorias lexicais em foco).

vi) Outros Lexemas Reais (OLR): Foi produzido um lexema existente em português, sem relação formal ou semântica com o estímulo (e.g. paga por desendividamento).

vii) Sem Resposta (SR).

Destas classes, resultam como formas agramaticais, sob o ponto de vista morfológico e semântico, os resultados classificados como Mutação Fonológica Parcial. Nos restantes casos, as formas devolvidas não são agramaticais, dado que semântica e morfologicamente estão bem construídas.

\subsection{Predições}

As predições que aqui expomos partem dos conceitos apresentados anteriormente na secção 1.2 e referem-se apenas ao confronto entre nomes contendo a série afixal -bil/-alizabilidade e outras unidades complexas heterocategoriais frequentes.

As correlações entre frequência, transparência semântica, saliência afixal, combinação entre afixos, complexidade morfológica dos padrões genolexicais, previsibilidade e a capacidade de construção de padrão suscitam as seguintes predições, tendo em conta que a transparência semântica pode ser avaliada pelas categorias de resultados Acerto Semântico Total, Alteração Semântica Parcial, Acerto Lexical Completo e Mutação Fonológica Parcial:

1. Quanto mais frequente for uma palavra, mais alta a sua previsibilidade e mais alta a sua perceção como semanticamente transparente.

Dado que a correlação entre transparência e previsibilidade não pode ser encarada apenas na sua atuação em palavras particulares, mas na combinação entre afixos,

2. quanto mais frequente for a combinação entre afixos integrada numa dada palavra, mais elevada a previsibilidade da combinação e mais elevada a perceção da mesma como transparente.

3. Quanto mais elevada a frequência da combinação entre as estruturas semânticas representadas pelos afixos, mais elevada é a perceção da palavra como transparente. Pelo 
contrário, uma combinação afixal pouco ou nada frequente resulta numa combinação semântica também ela pouco ou nada frequente, conduzindo a uma transparência semântica baixa.

4. A Mutação Fonológica Parcial é mais elevada quanto menos previsível for a combinação afixal. Logo, o Acerto Lexical Completo é mais baixo quanto menos previsível for a combinação afixal.

5. A Mutação Fonológica Parcial é mais elevada quanto menor for o poder de construção de padrão da combinação afixal. Logo, o Acerto Lexical Completo é mais baixo quanto menor for o poder de construção de padrão da combinação afixal.

\subsection{Tarefa de recordação}

A Figura 1 apresenta os resultados, arredondados à unidade, respeitantes à tarefa de recordação.

Os Acertos Lexicais Completos observaram-se em 95\% das recordações das palavras neutras. Segue-se a categoria de outras palavras complexas heterocategoriais frequentes (59\%), as palavras contendo a série -bil/-al-izabilidade (30\%) e as pseudopalavras (0\%).

A Mutação Fonológica Parcial ocorreu numa taxa mais elevada nas palavras em -bil/al-izabilidade (28\%), seguindo-se as pseudopalavras (25\%), as palavras heterocategoriais complexas frequentes $(15 \%)$ e as palavras neutras $(0 \%)$.

Não se verificou nenhum Acerto Semântico Total nas palavras em -bil/-al-izabilidade nem nas palavras heterocategoriais complexas frequentes. Apenas nas palavras neutras se obteve uma taxa de 1\% de resultados em Acerto Semântico Total. Esta categoria, como previsível, não ocorreu para as pseudopalavras.

A Alteração Semântica Parcial observou-se numa taxa mais elevada nas palavras em -bil/-al-izabilidade (35\%), seguindo-se as palavras heterocategoriais complexas frequentes $(13 \%)$ e as palavras neutras (1\%). Evidentemente, não se observou nenhum resultado desta categoria no caso das pseudopalavras $(0 \%)$.

O Desacerto na Categoria Lexical verificou-se em $1 \%$ das respostas de recordação das palavras em -bil/-al-izabilidade, não se tendo observado nas restantes categorias de palavras.

Outros Lexemas Reais obtiveram-se em 5\% das respostas relativas a outras palavras complexas heterocategoriais frequentes e em $1 \%$ das recordações das palavras neutras e em igual percentagem nas palavras em -bil/-al-izabilidade. 
A categoria Sem Resposta verificou-se em $75 \%$ das recordações das pseudopalavras, $7 \%$ das relativas às palavras complexas heterocategoriais frequentes, 5\% das relativas às palavras em -bil/-al-izabilidade e em 3\% para as palavras neutras.

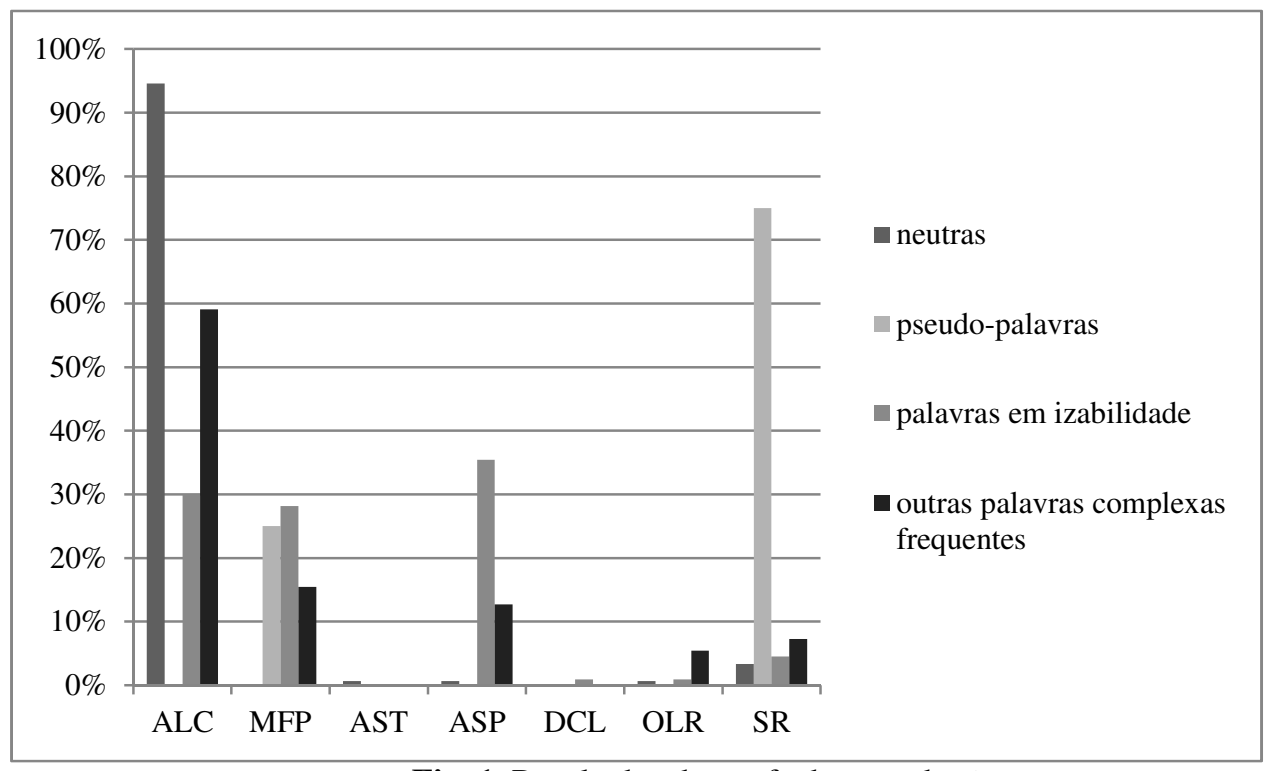

Fig. 1. Resultados da tarefa de recordação

Tanto nas palavras em -bil/-al-izabilidade quanto nos itens lexicais complexos heterocategoriais frequentes há a partilha da heterocategorialidade genolexical. A diferença que as separa reside no carácter de baixa frequência dos nomes em -bil/-al-izabilidade e no carácter mais frequente da outra categoria. $\mathrm{O}$ fator de frequência tem implicações que redundam nos resultados, como se verá:

-O Acerto Lexical Completo é mais elevado em outras palavras complexas, frequentes, do que nas palavras em -bil/-al-izabilidade, não frequentes, uma vez que a frequência determina a previsibilidade.

-A Mutação Fonológica Parcial é mais elevada nas palavras em -bil/-al-izabilidade do que na outra categoria, o que se justifica pelo carácter não frequente das primeiras. Seria necessária a existência de uma memorização, neste caso específico, da combinação de afixos, ou seja, do reconhecimento de um padrão genolexical, para que se obtivesse um acerto lexical completo. As respostas obtidas nas tarefas de recordação de palavras em -bil/-al-izabilidade demonstram dificuldade no reconhecimento dos constituintes morfológicos enquanto unidades 
com estruturas formal e semântica. Tal dificuldade enraíza-se na imprevisibilidade da combinação afixal em causa, uma vez que esta é não frequente. A imprevisibilidade da combinação afixal fundamenta a baixa saliência afixal.

Como exemplos de Mutação Fonológica Parcial, elencamos:

a) A substituição de componentes morfológicos por outros que não são operadores no mesmo padrão genolexical. Por exemplo, em vez de familiarizabilidade ocorreu famarialização. Neste nome, ainda que não coincidente com um item lexical do português, nem a sua presumida base coincidente com uma base do português, deu-se a intrusão do afixo -ção, operador na formação de nomes eventivos deverbais. O facto de o resultado não coincidir com lexemas reais ou potenciais do português leva a que não seja categorizado como mutação semântica.

b) Omissão de segmentos fonológicos não correspondentes com fronteiras morfológicas. Por exemplo, familiaribilidade por familiarizabilidade; comerçalizabidade/comercizibidade por comercializabilidade; contabilizalidade por contabilizabilidade. Esta situação também se verificou na categoria de outras palavras (desgovermentalização por desgovernamentalização; ormentação por ornamentalização). $\mathrm{O}$ contraste entre as fronteiras morfológicas, não comparecentes neste tipo de mutação, e as fronteiras silábicas, atuantes nesta mutação, mostram-se a seguir: (fronteiras morfológicas: famili-ar-iz-a-bil-idad-e; comerci-al-iz-a-bil-idad-e; conta-bil-iz-a-bil-idad-e; des-governament-al-iz-a-ção; orna-ment-al-iz-a-ção) vs. (fronteiras silábicas: fa.mi.li.a.ri.za.bi.li.da.de; co.mer.ci.a.li.za.bi.li.da.de; con.ta.bi.li.za.bi.li.da.de; des.go.ver.na.men.ta.li.za.ção; or.na.men.ta.li.za.ção).

c) Mudança de posição de um segmento dentro da sílaba ou entre sílabas (familirazidade por familiarizabilidade) ou mudança da posição da própria sílaba (funcionamentou por fundamentacionou).

d) Omissão de segmentos que correspondem a unidades morfológicas (familirazidade por familiarizabilidade; comercializidade por comerci-al-iz-a-bil-idad-e; legalizidade por legal-i-z-a-bil-idad-e).

e) Adição de segmentos fonológicos não contidos no estímulo (comerçaciobilidade por comercializabilidade). 
f) Substituição de segmentos fonológicos por outros iguais a segmentos ocorrentes noutras sílabas (alisibilidade por alisabilidade; analisibilidade por analisabilidade).

g) Adição de segmentos coincidentes com morfemas, provavelmente devido à influência de outros estímulos (ornamentabilizável por ornamentalizável).

- Não se verificou nenhum Acerto Semântico Total nem na recordação das palavras em -bil/-al-izabilidade nem na de outras palavras complexas. Isto deve-se ao facto de i) as alterações fonológicas não conduzirem à produção de item real ou potencial do português; ii) as alterações acarretarem ocorrências classificáveis como Alteração Semântica Parcial, tendo em conta que parte da estrutura semântica da palavra foi alterada.

- A Alteração Semântica Parcial é mais elevada nas palavras em -bil/-al-izabilidade do que nas outras palavras complexas. Tal deve-se ao facto de as primeiras terem tido uma taxa mais baixa de Acerto Lexical Completo. No caso das palavras em -bil/-al-izabilidade, verificou-se uma substituição dos estímulos por outras palavras reais ou potenciais do português que apresentam uma semelhança semântica forte com a semântica dos estímulos. Este é o caso de familiaridade por familiarizabilidade, contratualização, contratuabilidade, contratualidade por contratualizabilidade; comerciabilidade por comercializabilidade; globalização por globalizabilidade; contabilidade, contabilização por contabilizabilidade; materialidade por materializabilidade; sociabilidade, sociabilização por socializabilidade).

Dos resultados obtidos, observam-se duas situações relevantes para a compreensão do processamento de palavras complexas. São elas

i) a manutenção do sufixo -iz- e coocorrente inserção do sufixo -ção;

e

ii) a manutenção do sufixo -idade e perda do sufixo -iz-.

Estas duas situações advêm do facto de as palavras em -bil/-al-izabilidade não serem instâncias ou realizações de uma série afixal previsível, não coincidente com um padrão genolexical.

Assim, as duas soluções apontam que há uma tendência para os falantes seguirem padrões genolexicais previsíveis. Significa que as combinatórias que contêm i) -iz-ção e ii) idade sem -iz-, que correspondem a padrões mentais, são caracterizadas por uma capacidade de construção de padrão mais forte do que aquela apresentada pela combinação -bil/-alizabilidade. O padrão -iz-(a)-ção é no português contemporâneo muito forte. Nestes resultados 
trata-se de um padrão invocado pelo reconhecimento, ao nível do estímulo, do sufixo -iz-, que é saliente. Tal saliência conduz à recordação de um padrão não coincidente com a combinatória instanciada no estímulo, mas presente nas estruturas mentais do falante: o padrão de formação de nomes eventivos em -ção a partir de verbos em -iz-.

Este resultado sugere ter havido um efeito de previsão ao nível das estruturas morfológicas dentro da palavra. Tal efeito de previsão verifica-se quando a presença de um morfema ativa outro morfema, que, estando ausente do estímulo, se encontra associado ao primeiro no léxico mental.

Da mesma forma, o padrão de construção de nomes de qualidade através do sufixo idade possui igualmente uma forte capacidade de construção de padrão. Esta conclusão pode ser retirada da observação de que a recordação do sufixo -idade nos estímulos conduz ao abandono do sufixo -iz-. De facto, trata-se de uma associação afixal não frequente em português.

Outros Lexemas Reais foram encontrados para a recordação de palavras em -bil/-alizabilidade. Como exemplos, apresentamos durabilidade por utilizabilidade. Na recordação de outras palavras complexas surgiram, por exemplo, contabilização por compartimentalização, linguisticamente por egoisticamente, entre outros.

$\mathrm{O}$ facto de não ter havido uma taxa significativa de Sem Resposta para ambas as categorias de palavras indica que em ambas houve pelo menos alguns morfemas que foram identificados. Tal contrasta com a taxa de Sem Resposta obtida para as pseudopalavras, em que o reconhecimento de unidades morfológicas falha.

Das formas produzidas, a Tabela 5 mostra a relação entre formas gramaticais $(\mathrm{ALC}+\mathrm{ASP}+\mathrm{AST}+\mathrm{DCL}+\mathrm{OLR})$, formas agramaticais (MFP) e sem resposta (SR).

\begin{tabular}{|l|r|r|l|}
\hline & gramaticais & agramaticais & SR \\
\hline neutras & $96,6 \%$ & $0,0 \%$ & $3,4 \%$ \\
\hline pseudopalavras & $0,0 \%$ & $25,0 \%$ & $75,0 \%$ \\
\hline palavras em -izabilidade & $67,3 \%$ & $28,2 \%$ & $4,6 \%$ \\
\hline $\begin{array}{l}\text { outras palavras } \\
\text { complexas frequentes }\end{array}$ & $77,3 \%$ & $15,5 \%$ & $7,3 \%$ \\
\hline
\end{tabular}

Tabela 5. Relação entre formas gramaticais, agramaticais e sem resposta 
A Tabela 5 evidencia que a produção de formas gramaticais prevalece sobre as agramaticais nas palavras em -izabilidade, apesar de a sua percentagem ser inferior à que ocorre em outras palavras heterocategoriais. Este resultado deve-se ao facto de os falantes identificarem segmentos morfológicos em ambos os grupos de palavras, o que os leva a produções gramaticais, ainda que não coincidentes com a palavra-alvo. Por este motivo, é mais relevante operar com as 7 classes que desenhámos do que apenas com a dicotomia gramatical/agramatical.

\section{Discussão}

O nosso estudo, debruçado sobre a afixação, corrobora e expande os estudos de Bell \& Schäfer (2013; 2016), dedicados à composição. Segundo as autoras, não é apenas a transparência dos constituintes da palavra, mas também a relação semântica entre os mesmos que são responsáveis pela transparência semântica do lexema, havendo um peso da previsibilidade dessa relação no seu processamento.

A nossa metodologia integrou a análise de palavras que contêm a combinação afixal bil/-al-izabilidade, não frequente, em contraste com outras palavras contendo outras combinações afixais com carácter mais frequente, com o objetivo maior de determinar se essa combinação afixal corresponde a um padrão genolexical do português contemporâneo. A combinação obedece ao primeiro critério necessário ao estabelecimento do carácter de padrão: a obediência a constrangimentos estruturais. No entanto, não cumpre os requisitos processuais que resultam da interação entre frequência, previsibilidade face à combinação afixal, fatores que são determinantes para a transparência semântica e a saliência afixal, conducentes ao reconhecimento da combinação quer para a compreensão quer para a produção da palavra complexa.

O nosso estudo revela que a combinação afixal -bil/-al-izabilidade não constitui um padrão genolexical e que possui uma fraca capacidade de construção de padrão. A baixa frequência da combinatória determina a sua baixa previsibilidade e, logo, a sua fraca transparência semântica e a sua baixa saliência afixal. Resultados diferentes são observados nas outras palavras complexas heterocategoriais mais frequentes. Devido ao carácter frequente, esta segunda categoria mostra combinações afixais ou mesmo lexemas previsíveis. 
As experimentações sugerem ter ocorrido um efeito de previsão de elementos afixais sobre outros que, em conjunto com os primeiros, constituem padrões genolexicais do português, com carácter previsível. É este o caso do padrão de formação de nomes eventivos deverbais com a combinação -iz-(a)-ção e do padrão de formação de nomes de qualidade em -idade, que produz um efeito de omissão do sufixo -iz-. Estes dois padrões apresentam uma mais forte capacidade de construção de padrão do que a combinação em -bil/-al-iz(a)-bil-idade. Assim, a baixa frequência e a baixa aceitação demonstrada na tarefa de julgamento de aceitabilidade relativa a estas palavras tem relação com a inexistência de um padrão genolexical para esta combinatória. Como tal, não ocorre a identificação de morfemas necessária ao processamento de palavras complexas não frequentes devido ao não reconhecimento de um padrão correspondente à combinatória afixal específica. $\mathrm{O}$ não reconhecimento conduz a recategorizações de padrões previsíveis (-iz-(a)-ção e -idade sem -iz-). Os fatores em causa influenciam-se mutuamente: a baixa frequência conduz à baixa previsibilidade, à baixa transparência semântica e saliência afixal, que conduzem ao carácter de não padrão da combinatória. Em simultâneo, e seguindo Bell \& Schäfer (2016: 195), pelo facto de não se constituir como um padrão, os falantes tendem a não usar a combinatória.

As consequências para o tipo de processamento destas palavras são as seguintes:

De acordo com um modelo dual-route (Baayen et al., 1997; Schreuder \& Baayen, 1997), no processamento destas palavras, seria favorecida a via decomposicional, devido à baixa frequência das palavras e da combinatória e do seu carácter morfologicamente complexo (Niswander-Klement \& Pollatsek, 2006). No entanto, a baixa transparência semântica e a baixa saliência afixal obstaculizam essa análise, tendo em conta que, de acordo com Libben (2015), o objetivo da análise morfológica realizada pelo falante reside na criação de constructos conceptuais.

Contudo, o processamento holístico também sai desfavorecido, devido à baixa frequência das palavras e da combinatória, que não corresponde a combinatórias previsíveis pelo falante (Hawkins \& Blakeslee, 2004; Plag \& Baayen, 2009).

Os resultados para as palavras em -bil-/al-iz(a)-bil-idade contrastam com aqueles observados nas outras palavras complexas heterocategoriais mais frequentes contendo, por exemplo, a combinatória -ment-al-iz(a)-ção. Nesta categoria, destaca-se a frequência que 
conduz à previsibilidade, com consequências para o reconhecimento das mesmas (Schreuder \& Baayen, 1997).

O nosso estudo suporta o modelo multiple-route (Kuperman et al., 2010), segundo o qual a estrutura morfológica conglomera um conjunto de informações que têm um papel no processamento das palavras morfologicamente complexas, advindo essas informações dos morfemas, das combinações de morfemas, dos paradigmas morfológicos e da sua interação.

\section{Conclusão}

Este estudo partiu da observação empírica de palavras com a série afixal -bil-/al-iz(a)bil-idade. Trata-se de lexemas com uma frequência de 0/milhão de palavras, de acordo com o CRPC, e com ocorrências atestadas no Google e na imprensa (Público) em número muito baixo (563). Perante estes dados, procurámos determinar se estes objetos lexicais obedeceriam a constrangimentos estruturais, se seriam aceites pelos falantes nativos e se seriam processados da mesma forma que outras palavras complexas heterocategoriais mais frequentes. Ficou demonstrado neste trabalho que as palavras sob foco obedecem aos constrangimentos estruturais do português. Não obstante, as experiências de processamento mostram que as mesmas mostram baixa aceitabilidade por parte dos falantes nativos. As experiências mostram ainda que a série afixal -bil-/al-iz(a)-bil-idade não constitui um padrão genolexical.

\section{Referências}

Andrews, Sally (1986) Morphological influences on lexical access: Lexical or nonlexical effects? Journal of Memory and Language 25, pp. 726-740.

Andrews, Sally et al. (2004) Eye movements and morphological segmentation of compound words: There is a mouse in mousetrap. European Journal of Cognitive Psychology 16, pp. 285-311.

Aronoff, Mark (1976) Word-formation in generative grammar. Cambridge, MA: The MIT Press.

Baayen, Harald (1992) Quantitative aspects of morphological productivity. In Geert Booij e Jan van Marle (orgs.) Yearbook of Morphology 1991. Dordrecht: Kluwer, pp. 109-149.

Baayen, Harald (1993) On frequency, transparency and productivity. In Geert Booij e Jan van Marle (orgs.) Yearbook of Morphology 1992. Dordrecht: Kluwer, pp. 181-208. 
Baayen, Harald (2007) Storage and processing in the mental lexicon. In Gonia Jarema e Gary Libben (orgs.) The mental lexicon. Core perspectives. Amsterdam: Elsevier, pp.81-104.

Baayen, Harald et al. (1997) Singulars and plurals in Dutch: evidence for a parallel dual-route model. Journal of Memory and Language 37, pp. 94-117.

Baayen, Harald et al. (2007) Lexical dynamics for low-frequency complex words. A regression study across tasks and modalities. The mental lexicon 2 (3), pp. 419-463.

Baeskow, Heike (2015) Rules, patterns and schemata in word-formation. In Peter Müller, Ingeborg Ohnheiser, Susan Olsen e Franz Rainer (orgs.) Word-formation. An international handbook of the languages of Europe. Vol. 2. Berlin: Mouton de Gruyter, pp. 803-821.

Beauvillain, Cecile (1987) The integration of morphological and whole-word information during eye-fixations on prefixed and suffixed words. Journal of Memory and Language 35, pp. 801-820.

Bell, Melanie \& Martin Schäfer (2013) Semantic transparency: challenges for distributional semantics. In Aurelie Herbelot, Roberto Zamparelli e Gemma Boleda (orgs.) Proceedings of the IWCS 2013 workshop: Towards a formal distributional semantics. Potsdam: Association for Computational Linguistics (pp. 1-10).http://www.aclweb. org/anthology/W13-0601. Acedido a 23 de fevereiro de 2016.

Bell, Melanie \& Martin Schäfer (2016) Modelling semantic transparency. Morphology. http://link.springer.com/article/10.1007/s11525-016-9286-3. Acedido a 15 de maio de 2016.

Bertram, Raymond et al. (2000) The balance of storage and computation in morphological processing: the role of word formation type, affixal homonymy, and productivity. Journal of Experimental Psychology: Learning, Memory and Cognition 26, pp. 489511.

Booij, Geert (2010) Construction morphology. Oxford: Oxford University Press.

Burani, Cristina \& Alfonso Caramazza (1987) Representation and processing of derived words. Language and Cognitive Processes 2, pp. 217-227.

Butterworth, Brian (1993) Lexical representation. In Brian Butterworth (org.) Language production. Vol. 2. San Diego, CA: Academic Press, pp. 257-294. 
Caramazza, Alfonso et al. (1998) Lexical access and inflectional morphology. Cognition 28, pp. 297-332.

Corbin, Danielle (1987) Morphologie dérivationnelle et structuration du lexique. 2 vols. Tübingen: Niemeyer.

Frauenfelder, Ulrich Hans \& Robert Schreuder (1991) Constraining psycholinguistic models of morphological processing and representation: the role of productivity. In Geert Booij e Jan van Marle (orgs.), Yearbook of morphology 1990. Dordrecht: Kluwer, pp. 165-138.

Corpus de Referência do Português Contemporâneo (http://www.clul.ul.pt/pt/recursos/183reference-corpus-of-contemporary-portuguese-crpc)

Gaeta, Livio (2015) Restrictions in word-formation. In Peter Müller, Ingeborg Ohnheiser, Susan Olsen e Franz Rainer (orgs.) Word-formation. An international handbook of the languages of Europe. Vol. 2. Berlin: Mouton de Gruyter, pp. 859-875.

Giraudo, Hélène, \& Jonathan Grainger (2001) Priming complex words: evidence for supralexical representation of morphology. Psychonomic Bulletin \& Review 8, pp. 127131.

Halle, Morris (1973) Prolegomena to a theory of word formation. Linguistic Inquiry 4, pp. 316.

Hawkins, Jeff \& Sandra Blakeslee (2004) On intelligence. New York: Henry Holt and Company.

Ji, Hongbo et al. (2011) Benefits and costs of lexical decomposition and semantic integration during the processing of transparent and opaque English compounds. Journal of Memory and Language 65, pp. 406-430.

Kuperman, Victor et al. (2008) Morphological dynamics in compound processing. Language and Cognitive Processes 23, pp. 1089-1132.

Kuperman, Victor et al. (2010) Processing trade-offs in the reading of Dutch derived words. Journal of Memory and Language 62, pp. 83-97.

Kuperman, Victor et al. (2009) Reading polymorphemic Dutch compounds: toward a multiple route of lexical processing. Journal of Experimental Psychology: Human Perception and Performance 35, pp. 876-895.

Laudanna, Alessandro et al. (1994) Prefixes as processing units. Language and Cognitive Processes 9 (3), pp. 295-316. DOI:10.1080/01690969408402121. 
Lehtonen, Minna et al. (2007) Recognition of morphologically complex words in Finnish: evidence from event-related potentials. Brain Research 1148, pp. 123-137.

Libben, Gary (1998) Semantic transparency in the processing of compounds: consequences for representation, processing and impairment. Brain and Language 61, pp. 30-44.

Libben, Gary (2015) Word-formation in psycholinguistics and neurocognitive research. In Peter Müller, Ingeborg Ohnheiser, Susan Olsen e Franz Rainer (orgs.), Word-formation. An international handbook of the languages of Europe. Vol 1. Berlin: Mouton de Gruyter, pp. 203-217.

Libben, Gary et al. (2003) Compound fracture: The role of semantic transparency and morphological headedness. Brain and Language 84, pp. 50-64.

Linguateca (http://www.linguateca.pt)

Marslen-Wilson, William et al. (1994) Morphology and meaning in the English mental lexicon. Psychological Review 101 (1), pp. 3-33.

Niswander-Klement, Elizabeth \& Alexander Pollatsek (2006) The effects of root frequency, word frequency, and length on the processing of prefixed English words during reading. Memory and Cognition 34 (3), pp. 685-702.

Niswander, Elizabeth et al. (2000) The processing of derived and inflected words during reading. Language and Cognitive Processes 15, pp. 389-420.

Plag, Ingo, \& Harald Baayen (2009) Suffix ordering and morphological processing. Language 85 (1), pp. 109-152.

Rainer, Franz (2005) Constraints on productivity. In Peter Štekauer \& Rochelle Lieber (orgs.), Handbook of word-formation. Dordrecht: Springer, pp. 335-352.

Rio-Torto, Graça (1993) Formação de palavras em português. Aspectos da construção de avaliativos. Dissertação de Doutoramento em Linguística Portuguesa. Universidade de Coimbra.

Rio-Torto, Graça et al. (2016) Gramática derivacional do português. Coimbra: Imprensa da Universidade de Coimbra. 2. ${ }^{\mathrm{a}}$ ed.

Rodrigues, Alexandra Soares (2009) Portuguese converted deverbal nouns: constraints on their bases. Word Structure 2, pp. 69-107.

Rodrigues, Alexandra Soares (2014) Causative eventive chains and selection of affixes in Portuguese nominalisations. Lingue e Linguaggio XIII (1), pp. 159-184. 
Rodrigues, Alexandra Soares (2015) A gramática do léxico: léxico mental e morfologia derivacional. München: Lincom.

Rodrigues, Alexandra Soares (2016) O efeito da afixação pleonástica no processamento de lexemas morfologicamente complexos do português europeu. Comunicação apresentada ao Encontro Comemorativo dos 40 anos do CLUP. Porto, FLUP, novembro de 2016.

Rodrigues, Alexandra Soares (em publicação) Limits on the extension of affixal combination: structural restrictions and processing conditions. Suvremena Lingvistika.

Schreuder, Robert \& Harald Baayen (1995) Modeling morphological processing. In Laurie Beth Feldman (org.), Morphological aspects of language processing. Hillsdale, NJ: Erlbaum, pp. 345-364.

Schreuder, Robert \& Harald Baayen (1997) How complex simplex words can be. Journal of Memory and Language 37, pp. 118-139.

Schreuder, Robert et al. (2003) Parsing and semantic opacity. In Egbert Assink \& Dominiek Sandra (orgs.), Reading complex words. Cross-language studies. Dordrecht: Kluwer, pp. 159-189.

Taft, Marcus (1979) Lexical access via an orthographic code: the basic orthographic syllabic structure (BOSS). Journal of Verbal Learning and Verbal Behavior 18, pp. 21-39.

Taft, Marcus \& Kenneth Forster (1976) Lexical storage and retrieval of polymorphemic and polysyllabic words. Journal of Verbal Learning and Verbal Behavior 15, pp. 607-620. 